Article

\title{
Host-Targeted Antivirals Inhibit RACK1-mediated IRES Activities in HIV-1 Infection
}

\author{
Israa Malli, ${ }^{1,2}$ Namita Kumari, ${ }^{3,4}$ Larisa Dubrovsky, ${ }^{5}$ Andrey Ivanov, ${ }^{3}$ Michael Bukrinsky, ${ }^{5}$ Sergei \\ Nekhai, ${ }^{1,3,4}$ Sivanesan Dakshanamurthy, ${ }^{6 *}$ and Hemayet Ullah, ${ }^{2 *}$ \\ 1. Department of Microbiology, School of Medicine, Howard University, \\ Washington DC, USA; \\ 2. Department of Biology, College of Art and Science, Howard University, \\ Washington DC, USA; \\ 3. Center for Sickle Cell Disease, Howard University, Washington DC, USA; \\ 4. Department of Medicine, School of Medicine, Howard University, \\ Washington DC, USA; \\ 5. Department of Microbiology and Tropical Medicine, School of Medicine, \\ the George Washington University, \\ 1. Department of Oncology, and Clinical \& Experimental Therapeutics \\ Program, Lombardi Comprehensive Cancer Center, Georgetown University \\ Medical Center, Washington DC, USA. \\ * Correspondence: Sivanesan Dakshanamurthy (sd233@georgetown.edu) and Hemayet Ullah \\ (hullah@howard.edu)
}

\section{Abstract}

Host ribosome-associated scaffold protein Receptor for Activated C Kinase 1 (RACK1) is utilized by a diverse group of human viruses for Internal Ribosomal Entry Sites (IRES) - mediated translation of viral mRNAs. We recently reported inhibition of herpes virus by small molecules targeting the RACK1 functional site. Here, we tested these molecules against HIV-1 and HCV, as HIV-1 contains two potential IRES sites and HCV translation occurs exclusively through IRES. Compounds significantly downregulated activities of HIV-1- and HCV-related dicistronic reporter constructs in transfected HEK293T cells. The compounds also strongly downregulated production of the HIV-1 capsid protein p24 in HIV-infected cells, as well as production of HIV-1 Gag precursor p55 and p55derived proteins p24 and p17 in cells infected with the HIV-1 virus. Hepatitis C virus (HCV) IRES activities were also significantly inhibited by RACK1 inhibitor compounds. Since a number of human and plant pathogenic viruses are reported to use IRES, the RACK1 compounds can be established as broad host-targeted antivirals.

Keywords: RACK1; HIV-1; Hepatitis C; IRES; Translation; HEK293T; AZT; SD29; Arabidopsis; Host-Targeted Antiviral (HTA)

\section{Introduction}

Receptor for Activated C Kinase 1 (RACK1) identified in the 1990s is implicated in multiple cellular signaling and developmental pathways in both animal and plant kingdoms [1, 2]. RACK1 regulates diverse cellular functions serving as a scaffold protein and interacting with more than 150 different proteins either directly or as part of a larger complex [2, 3]. Mammalian 36-kDa RACK1 binds the active form of protein kinase C (PKC) and associates with the 40S ribosomal subunit near the mRNA exit channel to regulate translation [4-6]. RACK1 serves as a physical and functional link between PKC-mediated eukaryotic initiation factor 6 (eIF6) phosphorylation and subsequent ribosome activation [4,5]. Genetic depletion of RACK1 in many organisms including yeast [7], plants [8] and drosophila [9] is not lethal, but changes the sensitivity of small open reading frame translation and internal ribosome entry site (IRES) mediated translation $[9,10]$. This indicates that RACK1 may 
not be essential for general translation, but might regulate translation of specific mRNAs and may coordinate the interface between translational and regulatory networks [4].

As a scaffold protein, RACK1 is known to regulate many cellular processes such as cell proliferation, signaling, development, migration, and apoptosis (for review [1, 2]). In addition, RACK1 has increasingly been implicated in many viral, bacterial, fungal infections as well as in human diseases, including cancer, Alzheimer, Parkinson's, and alcohol addiction [9, 11-15]. Recent studies demonstrated that RACK1 regulates many pathogenic protozoan/microbial/fungal infections resulting in malaria [16], scarlet-like fever [11], and histoplasmosis [15]. In addition, RACK1 has been implicated in viral infections. RACK1 has recently been reported to induce apoptosis by interacting with VP5 protein of Infectious bursal disease virus (IBDV), that affects chickens and causes high fatality across the world [17]. RACK1 is essential for the replication of Porcine reproductive and respiratory syndrome virus, which is the major cause of economic loss for the swine industry worldwide [18]. RACK1 plays a role in the classical swine fever virus pathogenesis by interacting with the NS5A viral protein [19]. RACK1 also regulates the release of influenza A virus by interacting with the viral matrix protein M1 [20]. Finally, RACK1 regulates hepatitis B virus replication by counteracting the proapoptotic function of the core protein (HBc) [21].

However, a dominant role of RACK1 is emerging in viral pathogenesis, where it is utilized to aid a particular mode of viral mRNA translation within the host cells. To seize the host replication machinery, many viruses use the specialized RNA structural element, IRES, that can function independently of the 5' cap element [22]. IRESs were first reported in 1980s within the poliovirus and encephalomyocarditis virus genomes and subsequently found in cellular mRNAs as well [23-25]. Because IRES-utilizing viruses use this unique mechanism for translation, it has been hypothesized that specific inhibitors of IRES-dependent translation could serve as antivirals [26, 27]. To use IRESbased translation, viruses need host factors and host protein RACK1 has been established as a required key host factor in many IRES utilizing viral mRNA translations $[9,28]$. RACK1 has been found to be an essential determinant for hepatitis $\mathrm{C}$ virus (HCV) IRES-mediated translation and infection $[9,29]$. In Drosophila melanogaster, RACK1 acts as a cellular factor required for infection by IRES-containing picorna-like Drosophila C virus (DCV) [9]. The novel coronavirus that has created a global pandemic of epic proportion come from a family of viruses where use of IRES mode of translation has been reported [30]. Coronaviruses- Infectious bronchitis virus (IBV) and Mouse hepatitis virus (MHV) have been reported to use IRES elements to translate specific ORFs from their subgenomic mRNAs [31-33].

In HIV-1 infection, RACK1 was shown to interact with Nef, which binds to the carboxyl terminus of RACK1, allowing Nef to interact with PKC [34]. While most HIV-1 transcripts are translated in a cap-dependent manner, presence of IRES translation sites has been reported for HIV-1 isolates, and the RNA structure may facilitate the spread of the infection in the host under specific physiological conditions, such as cellular oxidative stress [35-38]. Thus, inhibition of IRES-based translation during HIV-1 infection may prevent successful infection of the host.

We previously solved the crystal structure of RACK1A from Arabidopsis Thaliana plant [9], which was then used to develop small molecules targeting RACK1's key tyrosine phosphorylation site. The Y248 phosphorylation has been shown to be a requirement for RACK1 protein's scaffolding function to mediate interaction with other proteins [39]. We recently showed the efficacy of these functional inhibitors against the herpes simplex virus proliferation, although the mechanism behind this inhibitory effect was not fully clarified [40]. In the current study, we used these compounds to investigate the role of RACK1 in IRES-mediated translation of HIV-1. Using dual-luciferase constructs, we tested the functional mechanism of the compounds' effect on HIV-1 and HCV IRESmediated translation. We demonstrated that RACK1 inhibitory compounds effectively inhibited IRES-dependent translation of HIV-1 and HCV. Based on our findings, we propose that RACK1 inhibitors can potentially be used as an anti-viral drug against a broad range of human diseasecausing IRES-using viruses including HIV-1 and HCV. 


\section{Materials and Methods}

\section{Cell culture}

Human epithelial type 2 (HEp-2) cells originated from human laryngeal carcinoma were a gift from Dr. Qiyi Tang (Howard University). HEp-2 cells were cultured at $5 \% \mathrm{CO}_{2}$ in Minimum Essential Medium Eagle (MEME) media contaning Earle's salts, L-glutamine and sodium bicarbonate (catalog \# M4655, Sigma-Aldrich Co. LLC) and supplemented with 10\% Fetal Bovine Serum (FBS), (Atlanta Biologicals, Cat\# S11150), 1\% Amphotericin B 250 g/mL (Fisher Bio-Reagents, Cat\# BP2645-50), and $1 \%$ Pen/Strep (100 U/ml penicillin and $100 \mu \mathrm{g} / \mathrm{ml}$ streptomycin) (ATCC® 30-2300 ${ }^{\mathrm{TM}}$ ).

\section{Antibodies}

Antibodies for actin (sc47778) were purchased from Santa Cruz. Antibodies that recognize HIV1 p55, p24 and 17 proteins were purchased from Abcam (ab63917). The RACK1 antibody (Cat\# sc17754) was obtained from the Santa Cruz Biotechnology (Dallas, TX).

\section{Plasmids and viruses}

HIV-1 proviral vector pNL4-3.Luc.R-E- (courtesy of Prof. Nathaniel Landau, NYU School of Medicine, New York, NY) and pHEF-VSVG expressing vector were obtained from the NIH AIDS Research and Reference Reagent Program. HIV-1(IIIB) strain was obtained from Advanced Biotechnologies. VSVG-pseudotyped HIV-1 virus was prepared as previously reported [41]. Plasmids with b-globin 5'UTR, HIV-1 gag leader, and HCV-IRES were a gift from Dr. Jeffry S. Kieft of the University of Colorado Denver [42]. Plasmids dI $\triangle E M C V$ and dI HIV-1-IRES were a gift from Dr. Marcelo López-Lastra of Pontifical Catholic University of Chile [43]. HIV-1(IIIB) strain was obtained from Advanced Biotechnologies.

\section{One round HIV-1 infection}

HEp2 cells were infected with VSVG-pseudotyped pNL4-3.Luc.R-E- virus. Cells were treated with RACK1 inhibitors $(1 \mu \mathrm{M}-100 \mu \mathrm{M})$ at $12 \mathrm{~h}$ post infection. Dimethyl sulfoxide (DMSO, Thermo Fisher Scientific Cat\# TS-20688) was used as vehicle control. After $48 \mathrm{~h}$ post treatment, luciferase activity was measured with Steady Lite Plus Reporter Gene Assay System (Perkin-Elmer) using GloMax®-Multi Microplate Multimode Reader (Promega).

\section{Bioluminescence Assays}

For the analysis of Renilla (RLUC) and Firefly luciferase (FLUC) activities from the dicistronic constructs, $80-90 \%$ confluent HEK293T cells were transfected with $200 \mathrm{ng}$ DNA of each of the indicated constructs using Lipofectamine 3000 (Thermo Fisher Cat\# L3000008) following manufacturer's protocol. After $12 \mathrm{~h}$, the media (MEM) were replaced with complete MEM supplemented with indicated compounds or DMSO vehicle and the cells were further cultured for 60 h. Duo-Luciferase Assay Kit (Genecopoeia, Cat\# LF004 and LF011) was used to measure the FLUC and RLUC activities from the same wells. Bioluminescence images were acquired by a Perkin Elmer IVIS Spectrum Imaging system immediately after adding luciferase substrate. Bioluminescence intensity in different wells was quantified in units of photons per second per centimeter squared per steradian $\left(\mathrm{p} / \mathrm{s} / \mathrm{cm}^{2} / \mathrm{sr}\right)$ by drawing a polygonal region of interest over the signals in images using Living Image 3.0 software (Caliper Life Sciences). For spectral unmixing analysis, guided spectral unmixing was first used on transfected cells only with the FLUC substrates or with RLUC substrates in separate wells and a series of luminescent images were acquired using emission filters at $500-700$ $\mathrm{nm}$, with $20 \mathrm{~nm}$ bandwidth. The acquired spectral data were then deconvoluted into its separate components. A library was created indicating the well images as either from FLUC or from RLUC (Living Image 3.0, Caliper Life Sciences). The relevant library spectra were then used to perform spectral unmixing on mixed RLUC and FLUC cells to separate the respective expressions from each of the luciferases. Data were exported to Excel and Prism 5 (Graphpad) for further analysis. 
Isolation and culture of PBMCs

Commercially obtained blood was diluted with PBS, mixed with Ficoll-Hypaque (Pharmacia) and centrifuged for $30 \mathrm{~min}$ at 2,100 rpm (800 g). Interphase was collected, cells were washed 3 times with PBS and then centrifuged $10 \mathrm{~min}$ at $1200 \mathrm{rpm}, 10 \mathrm{~min}$ at $1000 \mathrm{rpm}$ and $10 \mathrm{~min}$ at $800 \mathrm{rpm}$. PBMCs were resuspended at $2 \times 10^{6}$ cells per $1 \mathrm{ml}$ in RPMI complete medium supplemented with $10 \% \mathrm{FBS}$, $1 \%$ Pen/Strep, $1 \mathrm{mM}$ L-glutamine and rhIL-2 $(20 \mathrm{U} / \mathrm{ml})$. Cells were activated with PHA-P (Sigma) 5 $\mu \mathrm{g} / \mathrm{ml}$ for 2-3 days. Cells were washed twice with PBS prior to infection.

Preparation of monocyte-derived macrophages

PBMCs were resuspended at $8 \times 10^{6}$ cells/ml in RPMI with $10 \% \mathrm{HS}, 1 \%$ Pen/Strep, $1 \mathrm{mM} \mathrm{L-}$ glutamine. Monocytes were adhered to flask for 2 hours at $37^{\circ} \mathrm{C}$. PBS washed adherent cells were cultured in RPMI with 10\% HS, 1\% Pen/Strep, $1 \mathrm{mM}$ L-glutamine, supplemented with $2 \mathrm{ng} / \mathrm{ml}$ of MCSF (Sigma) in $\mathrm{CO}_{2}$ incubator for 24 hours. Cells were detached with $10 \mathrm{mM}$ EDTA, centrifuged and resuspended in RPMI complete medium with M-CSF to differentiate into macrophages at concentration $1 \times 10^{6}$ cells $/ \mathrm{ml}$ for one week. Cells were washed with PBS before infection.

Infection of monocyte-derived macrophages

Macrophages were treated with the indicated compounds $24 \mathrm{~h}$ prior to the infection and then infected in $200 \mu \mathrm{l}$ of medium in the presence of compounds using HIV-1 ADA (CCR5-tropic) virus at $5 \times 10^{5} \mathrm{cpm}$ of RT activity $/ 1 \times 10^{6}$ cells for 3 hours, followed by 3 washings with PBS. Infected cells were cultivated in fresh complete medium with the compounds. Every 3-4 days, half of the medium was changed.

\section{Cell lysate preparation and Western blot}

Cells were washed in PBS and lysed in $50 \mu \mathrm{l}$ M-PER Mammalian Protein Extraction Reagent (Thermo Scientific) supplemented with protease and phosphatase inhibitors.

Proteins were separated on Bio-Rad precast Criterion 4-12\% XT- Bis-Tris gels, transferred to nitrocellulose membranes (Amersham Inc., Piscataway, NJ, USA), and blocked with 5\% nonfat milk for $60 \mathrm{~min}$ at room temperature. Membranes were incubated overnight at $4^{\circ} \mathrm{C}$ with primary antibody, followed by incubation with a horseradish peroxidase-coupled secondary antibody and detection with enhanced chemiluminescence (Pierce, Rockford, IL, USA), according to standard methods.

Infection of CEM-T cells, PBMCs and PBL

PBMCs or PBL were activated with phytohemagglutinin (PHA) $(0.5 \mu \mathrm{g} / \mathrm{ml})$ for $48 \mathrm{~h}$ followed by interleukin (IL)-2 (10 U/ml) for $24 \mathrm{~h}$ prior to the infection. CEM-T cells, activated PBMCs or activated PBL were infected with T cell tropic HIV-1(IIIB) at a multiplicity of infection (MOI) of 0.01 infectious virus/target cell. Cells were treated with SD-12, SD-14 or SD-29 at indicated concentration at the time of infection. Cells were collected after 5 days for viral RNA analysis and supernatants were collected for p24 measurement.

\section{Determination of HIV-1 RNA.}

Total RNA was extracted using TRIzol reagent according to the manufacturer's protocol (Invitrogen, Grand Island, NY). Total RNA (100 ng) was reverse-transcribed to cDNA using Superscript ${ }^{\mathrm{TM}}$ RT-PCR kit (Invitrogen, Carlsbad, CA), hexamers and oligo-dT were used as primers. For real-time PCR analysis, cDNA was amplified using SYBR Green1 Master mix in Roche Light Cycler 480 (Roche Diagnostics, Indianapolis, IN). PCR was carried with denaturation at $95^{\circ} \mathrm{C}$ for 10 seconds, annealing at $60^{\circ} \mathrm{C}$ for 10 seconds, and extension at $72^{\circ} \mathrm{C}$ for 10 seconds for 45 cycles. The $18 \mathrm{~S}$ rRNA was used as a house keeping normalization standard for quantification of mRNA levels of HIV-1 gag. Primer sequences for HIV gag, forward-ATAATCCACCTATCCCAGTAGGAGAAAT, reverse- TITGGTCCITGTCITATGTCCAGAATGC and 18SrRNA, forward- 
CTGTTGCTACATCGACCTTT, reverse- CTCCAGGTTTTGCAACCAGT. Mean Cp values for target genes and $18 \mathrm{~S}$ rRNA were determined and $\triangle \Delta \mathrm{Ct}$ method was used to calculate relative expression levels.

\section{Statistical Analysis and Calculations.}

All assays were run in triplicates unless as indicated to ensure reproducibility of the data. All data are presented as a mean \pm Standard Error of the Mean (SEM) from all experiments.

\section{Results}

\section{RACK1 inhibitors regulate IRES-mediated translation in HEK293T cells}

Bicistronic reporter assays have been the most commonly used technique for IRES-based translation activities, and we utilized a series of dual-luciferase reporter constructs to determine if previuosly identified RACK1-inihibitor compounds affect IRES-mediated translation of HIV-1 gag ( $5^{\prime}$ UTR 1-384 nts) and dl HIV-1 IRES (5'UTR of the HIV-1 full-length mRNA) constructs [42, 43] in HEK293T cells (Fig. 1A). Dual vectors express mRNA coding Renilla luciferase (RLUC) and firefly luciferase (FLUC) under control of SV40 promoter. RLUC translation was dependent on the $5^{\prime}$ cap of the mRNA, whereas FLUC translation was dependent on the inserted HIV-1 IRES or HCV IRES, which was used as a positive control for IRES activities (Fig. 1A). We also used a dual construct with $\beta$-globin 5' UTR instead of IRES as a negative control for the HIV-1 gag IRES, while the dl $\triangle \mathrm{EMCV}$ vector containing the defective EMCV IRES inserted upstream of the FLUC reporter was used as negative control for IRES activity from the dl-HIV-1 IRES constructs (Fig. 1A). The cells were transfected with the corresponding dual-luciferase reporter plasmids and treated with RACK1inhibitor compounds SD-29 or SD29-14 at different concentrations $(1 \mu \mathrm{M}, 10 \mu \mathrm{M}$ and $50 \mu \mathrm{M})$. The IRES activities were measured by calculating the ratio of FLUC to RLUC bioluminescence signals [42]. Figure 1B shows representative FLUC (green) and RLUC (red) signals obtained from the cells after spectral unmixing of the two signals from the same wells. The DMSO treated cells transfected with the IRES from HIV-1 produced primarily FLUC (green) signal, however, spectral mixing with the basal level of RLUC (red) signals produced slightly yellowish signal emanating from the combination of green and red signals (Fig. 1B). Treatment with SD-29 or SD-29-14 significantly inhibited FLUC (green) signal but had no effect on the basal RLUC (red) signal that was driven by the 5'-cap dependent translation (Fig. 1B). When quantified, both SD-29 and SD-29-14 had strong inhibitory effects on both HIV-1 IRES constructs as shown in Fig. 1C. A positive control for the IRES activities, HCV IRES containing bicistronic construct (Fig. 1C middle panel), showed the highest level of IRES based FLUC (green) signal in DMSO treated cells compared to all other constructs, thereby providing high confidence in the results obtained with the HIV-1 IRES constructs. The HCV IRES based expression was significantly inhibited by the RACK1 inhibitor compound SD 29-14 as indicated by the lack of any visible FLUC (green) signal (Figs. 1B and 1C). The compound SD29 was somewhat less effective compared to SD29-14, as it only reduced the HIV-1 IRES-driven but not the HCV IRESdriven translation.

As a negative control, the IRES sequences were replaced with $5^{\prime}$ UTR sequences of $\beta$-globin gene or a defective EMCV IRES inserted upstream of the FLUC reporter. As the reporter FLUC expression from these control constructs was barely detectable, the spectral unmixing assay was not performed. Instead, the average relative radiance from the FLUC and RLUC constructs was quantified and reported in Table I. As can be seen in Table I, both of the negative control constructs showed only negligible level of IRES activities which was not affected by the treatment with the compounds. Considering the robust activities in the positive control and almost no signals from the negative controls, it can be safely concluded that the RACK1 inhibitor compounds have specifically inhibited the HIV-1 IRES activities. As the HIV-1 IRES activities have been reported to increase in various physiological conditions including in the oxidative stress generated due to the infection [37], the effective performance of the small compounds in inhibiting the HIV-1 IRES activities indicates that the development of these compounds will be useful in combating HIV-1 IRES based translations. 
A

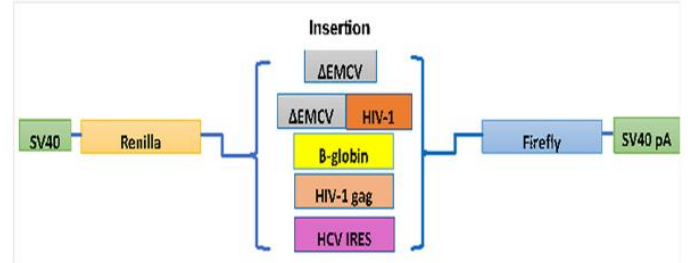

B

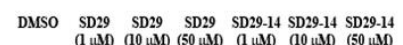
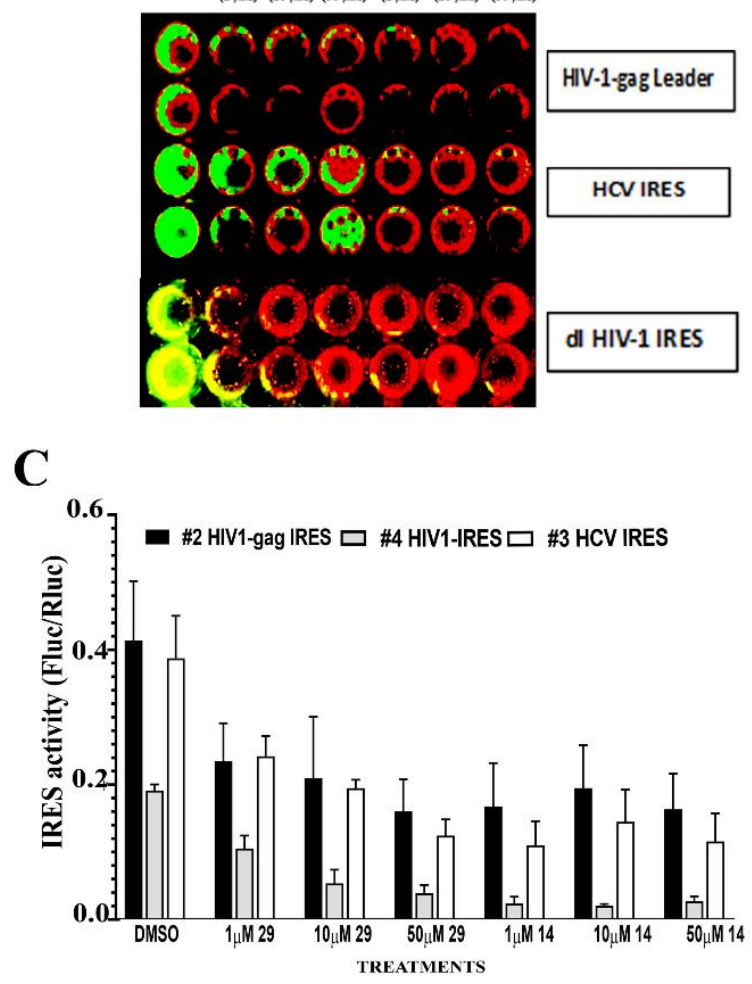

Figure I

Figure 1. The RACK1 inhibitor compounds prevent HIV-1 and HCV IRES activities (A) Schematic representation of the dual luciferase bicistronic constructs that express RLUC from the SV40 promoter. (B) The dl $\triangle$ EMCV [43] , dl HIV-1 IRES [43], HIV1-gag Leader IRES [38], HCV IRES [38], 5' UTR DNA sequences from $\beta$-globin vectors (100 ng/well) were transfected into HEK293T cells using master-mix of the plasmids with lipofectamine 3000 reagents. After 12 hrs of incubation, compounds were added and incubated for additional $60 \mathrm{hrs}$. Luciferase activity was assayed with IVIS Spectrum machine using spectral unmixing option for RLUC and FLUC luminescence. (C) Luminescence for FLUC and RLUC (Avg Radiance $[\mathrm{p} / \mathrm{s} / \mathrm{cm} \leq / \mathrm{sr}]$ ) were used to determine the IRES activities (FLUC/RLUC). Values are the mean (+/- SEM) for two replicates as depicted. 


\section{RACK1 inhibitor compounds regulate IRES activities in HEp-2 cells as well}

In order to have confidence in the results depicted in Fig. 1B, we utilized a different HIV-1 permissive cell line, HEp-2, to investigate whether the compounds can inhibit the IRES activities from the same constructs as depicted in Fig. 1A. The Dual-luciferase reporter constructs were used to determine if the RACK1 functional inhibitor compounds target IRES-mediated translation. The HEp2 cells transfected with the indicated plasmids were treated with one of the RACK1 inhibitors (SD29) for a confirmatory study. Here the IRES activity was measured by calculating the ratio of raw luminescense data from FLUC to raw data from RLUC and presented in Table 2. Results show that, like in the HEK293T cells, in the HEp-2 cells, the SD29 compound was also able to inhibit the HIV-1 IRES activities from both of the constructs (Table 2). The positive control, HCV IRES, also showed a very high activity when the compound was not present, but almost $50 \%$ reduction can be seen when the compound was present. In the negative control cells with beta-globin and with $\triangle E M C V$ plasmid, almost all of the signals, as expected, were coming from the cap-dependent renilla bioluminescence,

Table 1. Raw luciferase values from control construct transfections (Average Radiances)

\begin{tabular}{|c|c|c|c|}
\hline IRES ( $\beta$-Globin) & FLUC $\left(\mathrm{p} / \mathrm{s} / \mathrm{cm}^{2} / \mathrm{sr}\right)$ & RLUC (p/s/cm²/sr) & FLUC/RLUC Ratio \\
\hline DMSO & $5.51 E+04$ & $1.02 \mathrm{E}+06$ & $0.055 \pm 0.001$ \\
\hline $\operatorname{SD} 29(1 \mu \mathrm{M})$ & $4.02 E+04$ & $5.82 \mathrm{E}+05$ & $0.068 \pm 0.002$ \\
\hline SD29 $(10 \mu \mathrm{M})$ & $3.52 E+04$ & 4.70E+05 & $0.074 \pm 0.006$ \\
\hline $\mathrm{SD} 29(50 \mu \mathrm{M})$ & $3.74 \mathrm{E}+04$ & $4.01 \mathrm{E}+05$ & $0.093 \pm 0.004$ \\
\hline SD29-14 (1 $\mu \mathrm{M})$ & $2.21 E+04$ & $3.29 \mathrm{E}+05$ & $0.067 \pm 0.002$ \\
\hline SD29-14 (10 $\mu \mathrm{M})$ & $2.72 E+04$ & $4.39 E+05$ & $0.062 \pm 0.003$ \\
\hline SD29-14 $(50 \mu \mathrm{M})$ & $1.74 \mathrm{E}+04$ & $2.62 \mathrm{E}+05$ & $0.067 \pm 0.004$ \\
\hline $\operatorname{IRES}(\triangle \mathrm{EMCV})$ & FLUC $\left(\mathrm{p} / \mathrm{s} / \mathrm{cm}^{2} / \mathrm{sr}\right)$ & RLUC (p/s/cm²/sr) & FLUC/RLUC Ratio \\
\hline DMSO & $4.21 E+04$ & $9.68 \mathrm{E}+05$ & $0.044 \pm 0.003$ \\
\hline $\operatorname{SD} 29(1 \mu \mathrm{M})$ & $1.66 \mathrm{E}+04$ & $2.49 \mathrm{E}+05$ & $0.067 \pm 0.002$ \\
\hline SD29 $(10 \mu \mathrm{M})$ & $1.17 \mathrm{E}+04$ & $1.36 \mathrm{E}+05$ & $0.086 \pm 0.003$ \\
\hline $\mathrm{SD} 29(50 \mu \mathrm{M})$ & $1.54 \mathrm{E}+04$ & $2.25 \mathrm{E}+05$ & $0.068 \pm 0.003$ \\
\hline SD29-14 (1 $\mu \mathrm{M})$ & $2.88 \mathrm{E}+04$ & $4.75 E+05$ & $0.060 \pm 0.002$ \\
\hline SD29-14 (10 $\mu \mathrm{M})$ & $1.32 \mathrm{E}+04$ & $1.90 \mathrm{E}+05$ & $0.070 \pm 0.000$ \\
\hline SD29-14 (50 $\mu \mathrm{M})$ & $1.59 \mathrm{E}+04$ & $2.46 \mathrm{E}+05$ & $0.065 \pm 0.002$ \\
\hline
\end{tabular}

Raw values for the FLUC and RLUC are the average radiances from three replicates. Values for the FLUC/RLUC ratio are the average of three replicates \pm SEM

while the IRES-dependent firefly signal appeared at the background level. These results in another cell line confirm that the RACK1 functional inhibitor compounds can specifically inhibit HIV-1 and HCV IRES activities. 
Table 2. Inhibition of HIV-1 and HCV IRES activities by RACK1-inhibitor compound in HEp-2 cells.

\begin{tabular}{|c|c|c|c|c|}
\hline & & FLUC $\left(\mathrm{p} / \mathrm{s} / \mathrm{cm}^{2} / \mathrm{sr}\right)$ & $\operatorname{RLUC}\left(\mathrm{p} / \mathrm{s} / \mathrm{cm}^{2} / \mathrm{sr}\right)$ & Ratio \\
\hline \multirow[t]{2}{*}{ IRES ( $\beta$-Globin) } & DMSO & 542.026 & 4833.1 & $0.112 \pm 0.0035$ \\
\hline & SD29 $100 \mu \mathrm{M}$ & 430.005 & 3496.29 & $0.123 \pm 0.0044$ \\
\hline \multirow{2}{*}{$\begin{array}{l}\text { IRES (HIV-1 gag } \\
\text { Leader) }\end{array}$} & DMSO & 3488.57 & 13310.3 & $0.262 \pm 0.0032$ \\
\hline & SD29 $100 \mu \mathrm{M}$ & 2037.2 & 14984.5 & $0.136 \pm 0.0017$ \\
\hline \multirow[t]{2}{*}{ IRES (HCV) } & DMSO & 63016 & 61586 & $1.023 \pm 0.004$ \\
\hline & SD29 $100 \mu \mathrm{M}$ & 30506 & 50906 & $0.600 \pm 0.002$ \\
\hline \multirow[t]{2}{*}{ IRES (HIV-1) } & DMSO & 63016 & 61586 & $1.023 \pm 0.004$ \\
\hline & SD29 $100 \mu \mathrm{M}$ & 30506 & 50906 & $0.600 \pm 0.002$ \\
\hline \multirow[t]{2}{*}{ IRES ( $\triangle$ EMCV) } & DMSO & 238.003 & 20333.4 & $0.012 \pm 0.005$ \\
\hline & SD29 $100 \mu \mathrm{M}$ & 210.002 & 19173.95 & $0.011 \pm 0.0032$ \\
\hline
\end{tabular}

Values for the FLUC/RLUC ratio are from the average of three replicates \pm SEM

RACK1-inhibitor compounds prevented pseudotyped HIV-1 infection in HEp2 cells

To further evaluate the effect of SD29 on HIV replication, HEp-2 cells were infected with VSVG-pseudotyped pNL4-3.Luc.R-E- virus and treated with different concentrations of SD-29, SD29-12, DMSO, or left untreated. The cells were cultured for $48 \mathrm{~h}$, and then luciferase activity was measured. Treatment with SD-29 and with SD29-12 significantly reduced one round HIV-1 infection (Fig. 2A). Lysates of the treated samples were used to investigate whether the compound treatment had any inhibitory effect on RACK1 expression. As can be seen, in both SD29 and SD29-12 treated samples the RACK1 expression was significantly downregulated as compared with lysates treated with DMSO (Fig. 2B). There were not any significant effect seen on the viabilities of the cells from the treatments as reported earlier [40]. 
A

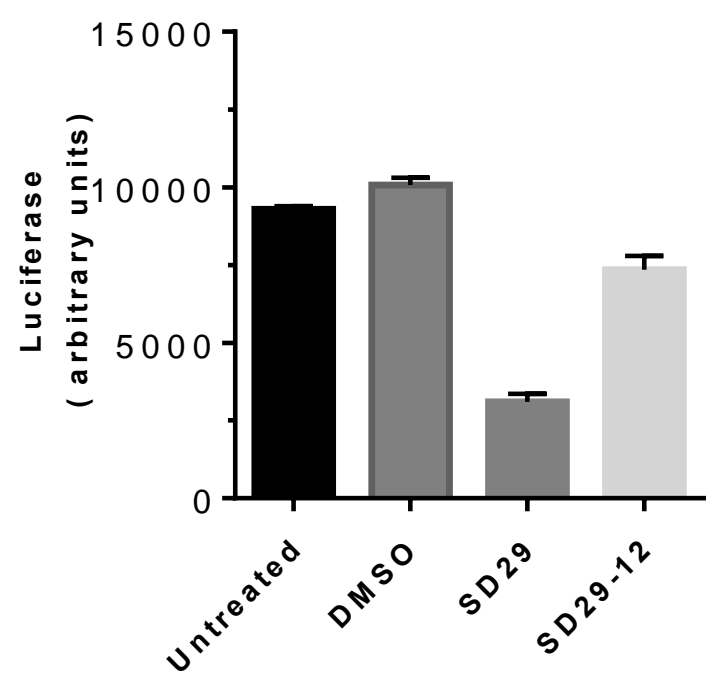

B

SD29 SD29-12 No

DMSO 100 uM 100 uM Infection

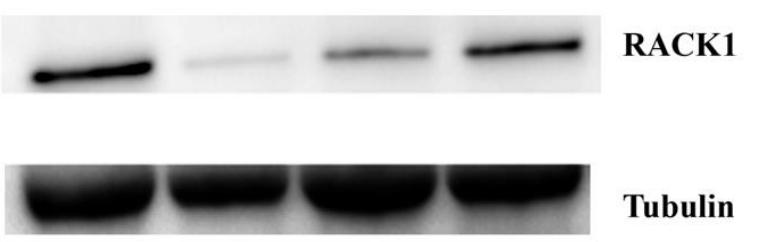

Figure 2. Evaluation of the effect of RACK1-targeting compounds on pseudotyped HIV-1 replication in Hep-2 cells. (A) Luciferase signals from transfected HEp-2 cells with infected with a single round of VSV-G-pseudotyped pNL4-3. Luc. R-E- virus and 48 hours of incubation with RACK1-targeting small molecules: (SD29 $100 \mu \mathrm{M}$ and SD29-12 $100 \mu \mathrm{M}$ ) or DMSO, as vehicle control or no treatment. (B) HEp-2 cell lysates after 48 hours incubation with pesudotyped HIV-1 virus in the presence of the indicated RACK1 inhibitor compounds or vehicle DMSO were blotted with antiRACK1 antibody (Upper panel), Tubulin antibody was used to probe the same membrane for loading control (lower panel).

RACK1 inhibitors SD29, SD-12 and SD-14 prevented live HIV-1 replication in cultured T cells and PBMCs

The effects of RACK1 inhibitors SD29, SD-12 and SD-14 on HIV-1 replication were analyzed in CEM T cells infected with HIV-1(III). Gag p55 (Fig. 3A) and p24 (Fig. 3B) were measured as an indicator of HIV-1 replication. HIV-1 replication was inhibited by $10 \mu \mathrm{M}$ concentration of SD29, SD12 and SD-14 but not by DMSO. Further, SD29 inhibited HIV-1 replication at lower concentration of $1 \mu \mathrm{M}$. We also analyzed the effects of RACK1 inhibitors SD29, SD-12 and SD-14 on HIV-1replication in PBMCs. The PBMCs were activated with PHA and IL-2 (described in Materials and Methods) and then infected with HIV-1(III). As shown in Figs. 3C and 3D, SD29, SD-12 and SD-14 markedly inhibited HIV-1 replication at $10 \mu \mathrm{M}$. AZT at $25 \mu \mathrm{M}$ shows similar inhibition. Thus, taken together, these results indicate that RACK1 inhibitors SD29, SD-12 and SD-14 markedly inhibit HIV-1 infection of CEM-T cells and PBMCs. 
A

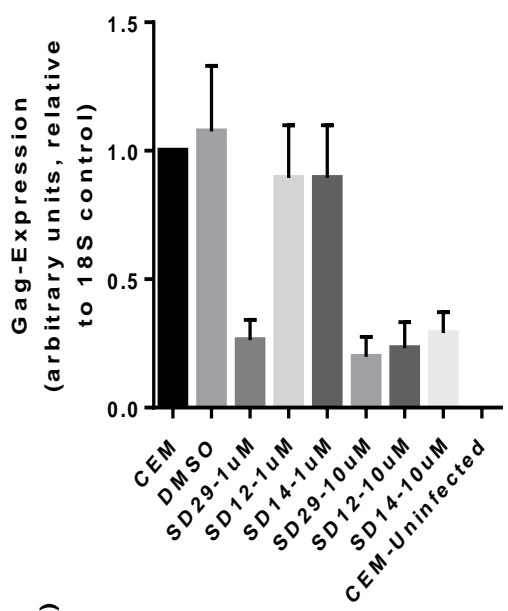

C

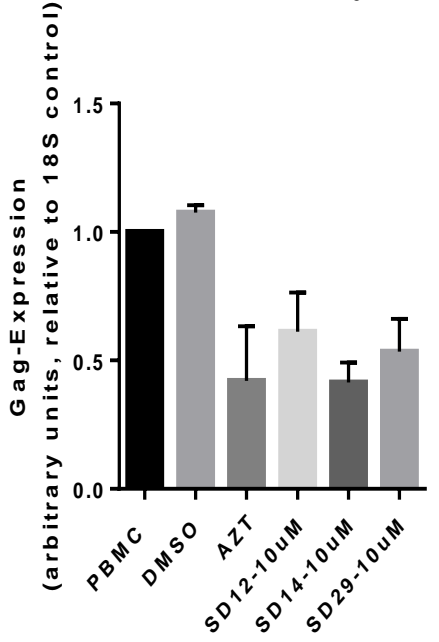

B

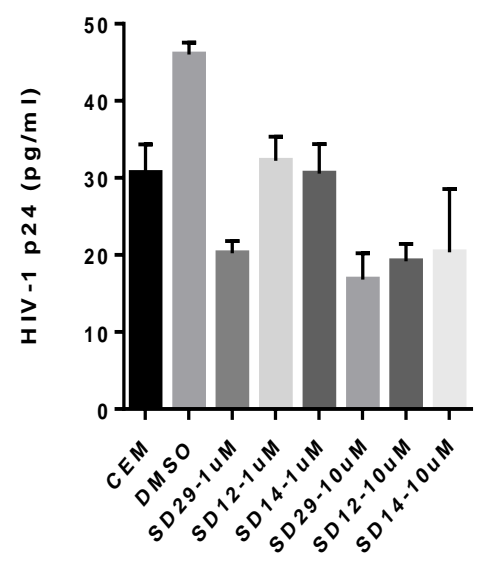

D

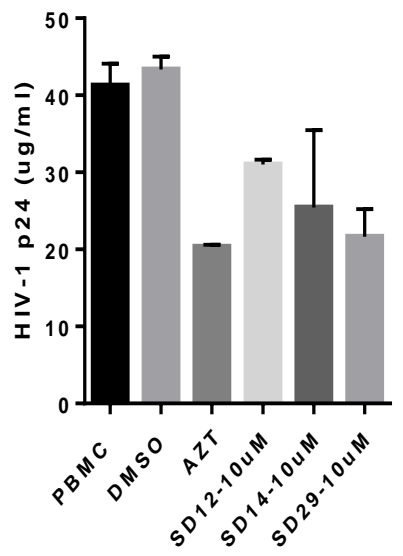

Figure 3. Inhibition of HIV-1(IIIB) replication in CEM T cells and PBMCs. (A) CEM T cells (A\&B) were infected with HIV-1(IIB) virus at an MOI of 0.01 and treated with the indicated concentrations of RACK1 inhibitors SD29, SD-12 or SD-14, and incubated at $37^{\circ} \mathrm{C}$. Five days post-infection cells were harvested for RNA extraction and subsequent quantitative real-time RT-PCR with gag-specific primers (A). Culture supernatants were collected and p24 was measured by ELISA (B). (C\&D) PBMCs activated with PHA and IL-2 were infected with HIV-1(IIIB) virus at an MOI of 0.01 and treated with 10uM RACK1 inhibitors SD29, SD-12 or SD-14 and AZT 25uM and incubated at $37^{\circ} \mathrm{C}$. Five days postinfection cells were harvested for RNA extraction and subsequent quantitative real-time RT-PCR with gag-specific primers(C). Culture supernatants were collected and p24 was measured by ELISA (D).

RACK1-inhibitor compounds inhibit HIV-1 ADA replication in macrophages

In order to investigate whether the inhibition of Gag precursor IRES activities by the compounds results in the down regulation of the Gag precursor and the protein products, monocyte-derived macrophages were infected with the HIV-1 ADA strain for 9 days. The resultant cell lysates were probed for the expression of the Gag precursor (p55) and the p24 and p17 proteins using a rabbit polyclonal HIV-1 p55 + p24 + p17 antibodies. The experiment also tested the dose response, hence, a concentration of $0.1 \mu \mathrm{M}$ to $100 \mu \mathrm{M}$ treatment series were used. As can be seen in Fig. 4, the treatment of the cells with SD-29 significantly reduced the abundance of HIV-1 Gag precursors and the resultant proteins in infected macrophages. The assay showed that the reduction of the p55 and p24 was significant compared to the DMSO treated samples. The corresponding downregulation of the RACK1 protein expression in the same samples indicated that it is possible that the RACK1 expression is needed for the synthesis of the Gag proteins from their precursor (Fig. 4B). Though the pattern of the inhibition in the Gag proteins does not indicate that the treatment is producing immature viral particles, it will be worthwhile in the future to test the infectivity of such viral particles to assess the implication of this result. 

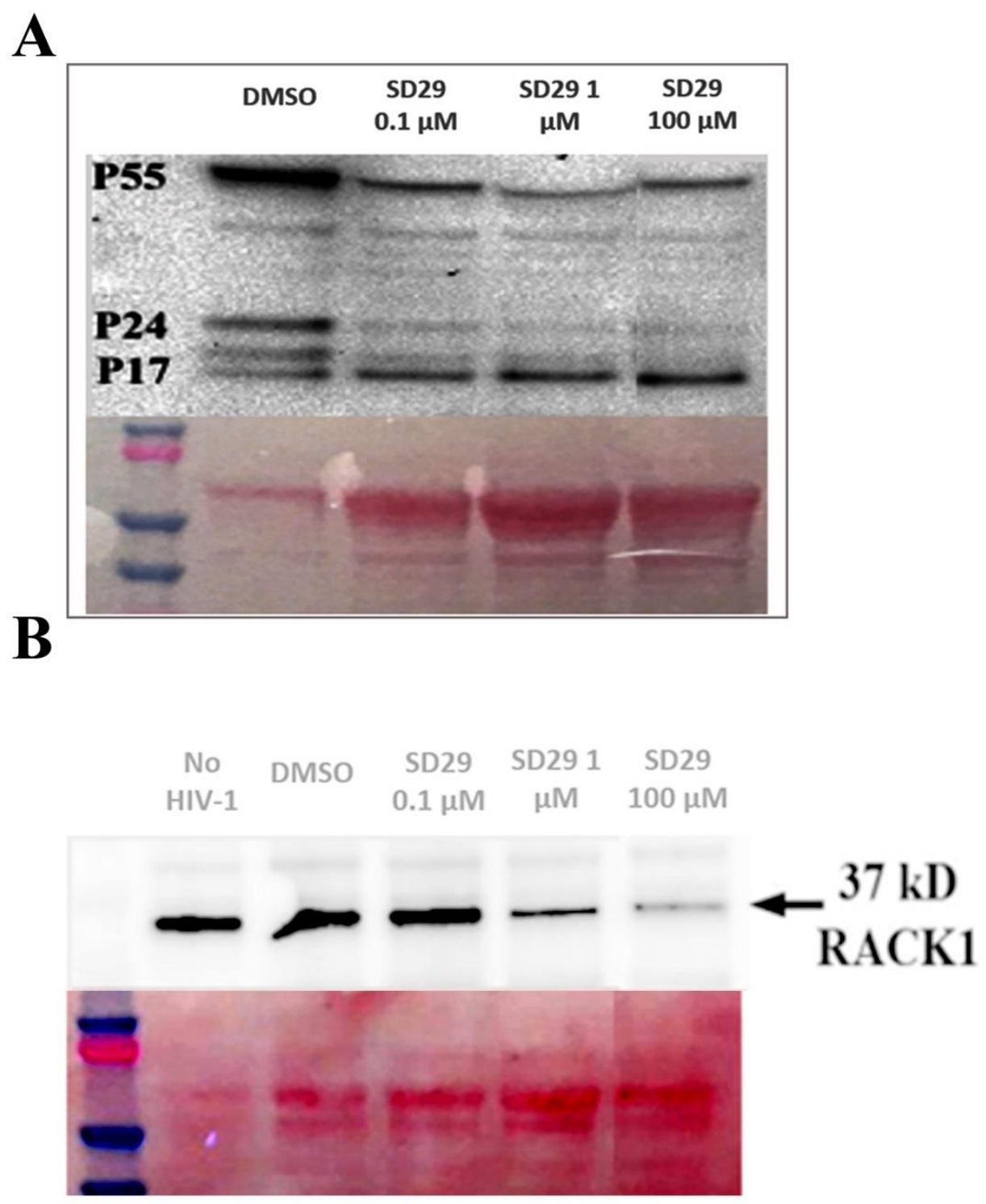

Figure 4. RACK1 functional inhibitor compounds inhibit HIV-1 ADA strain Gag precursor/protein expression. Lysates from nine-day long treated macrophages with the indicated concentration of compound SD29 were probed with a rabbit polyclonal HIV1 p55 + p24 + p17 antibody (Cat\#ab63917) from Abcam (Cambridge, MA). Compounds were dissolved in DMSO. The Gag precursor band (p55) and the processed p24 and p17 protein bands are labeled in the upper panel. The no virus control did not show any bands reacting with the antibody. The lower panel shows the same membrane stained with ponceau dye before probing with the antibody to show as the loading control. Repeated water rinses were used to remove the ponceau stains and then blocked with $5 \%$ dry milk (Bio-Rad) before probing with the antibody (A). This panel shows the same lysates (including a no virus control) probed with an anti-RACK1 antibody. Ponceau stain was used in the same manner as described in panel $\mathrm{A}$ as the loading control. Note that a lane between $1 \mathrm{uM}$ and $100 \mathrm{uM}$ has been removed due to loading anomaly (B).

Structural basis of the role of RACK1-targeting compounds in IRES-mediated antiviral activity. Earlier studies by our group and others described the involvement of the IRES element in the HIV-1 infection [40, 44-47]. Further, Locker et al reported that eIF3 and the small ribosomal subunit 
bind HIV RNA within gag open reading frame and they proposed the formation of a ternary eIF340S- HIV-1 IRES complex [45]. Moreover, a number of X-ray and cryo-EM structures reported the formation of the ternary structure of the eIF3-40S-HCV IRES[48-50]. Intrigued by these studies, we sought to obtain a structural basis on the role of RACK1 in the IRES-based anti-viral activity, and developed a hypothetical structural model of RACK1-eIF3-40S-HIV IRES model based on the structure of RACK1-eIF3-40S-HCV IRES[48-50]. The structural model of RACK1-eIF3-40S IRES complex (Fig. 5) was developed using the following procedure: the RACK1 structure was fitted to the mammalian 40S HCV-IRES complex (pdb: 5flx). Then we fitted the obtained RACK1-40S- HCV IRES1 structural model with the crystalized elf3-43S complex structure (pdb: 5a5u and 5a5t) and eIF3RACK1-40S model described by des Georges et al [51]. Thus, we developed the final structural model of the elf3-RACK1-40S IRES complex (Fig. 5).

The structural model obtained suggests that RACK1 is located sterically but not in direct amino acid residue contact with the IRES (Fig. 5). Distinctly, Rack1 is in direct contact with peripheral domain of the translation initiation factor eIF3 (Fig. 5). Based on this hypothetical model, we speculate that our RACK1 SD29 inhibitor compounds have the potential to block the functional interaction of eIF3 with RACK1 as well as likely disturb the indirect contact of IRES with Rack1. We think that binding of our small molecules to RACK1 blocks the IRES functional activity in two ways 1) blockade in the front door, by blocking the eIF3 interaction with RACK1 (a functional link between RACK1 and IRES-dependent translation); 2) blockade in the back door, via binding to RACK1 and triggering conformational changes that render RACK1 unable to interact with the viral IRES.

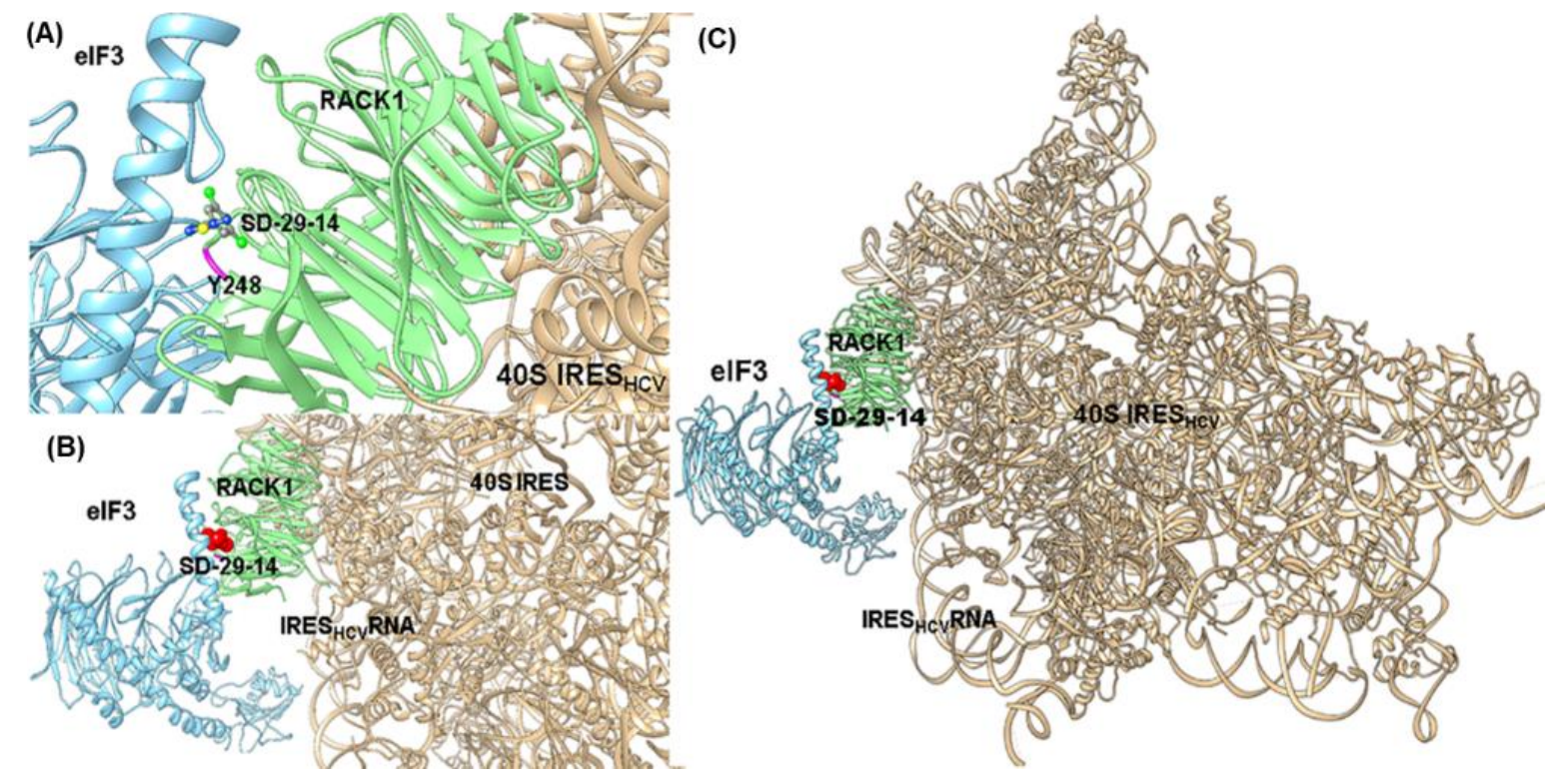

Figure 5. Structural model of Rack1-elf3-40S IRES complex . A) close up view of the model shown as protein ribbon model with eIF3 (blue), Rack1 (green), Rack1 small molecule inhibitor SD-29-14 shown in ball \& stick model (colored by atom type), Y238 phosphorylation site (magenta), 40S IRES (brown) B) close view with full eIF3 structure is shown with SD-29-14 shown as surface model (red) blocking the interaction between Rack1 and eIF3 C) full view of the model Rack1-elf3-40S IRES complex. 


\section{Discussion}

Due to the small genome size and rapid replication, viruses are inherently very apt at accumulating mutations rapidly to overcome sustained inhibitory effect from drugs that target the virus directly. In this regard, targeting a host-factor essential for viral replication can be an attractive alternative to develop durable anti-viral drugs. Previously we developed a host factor RACK1targeting broad antiviral compound showing efficacy against herpes-simplex virus-1, but whether the RACK1 inhibitor compounds affect viral IRES activities has not been addressed before [40]. Here we report inhibition of HIV-1 proliferation by RACK1-inhibior compounds that prevented IRESmediated cap-independent translation from Gag gene of HIV-1. The compounds were equally competent in inhibiting IRES activities from an HCV bicistronic construct as well. In addition, we report that the RACK1-targeting compounds inhibited one round and multiple rounds of HIV-1 replication.

HIV-1 utilizes IRES in a cell type-specific replication [42] whereby HIV-1 mRNAs bind to the $40 S$ ribosomal subunit via one of two IRESs and recruit eIF-3 [52]. To evaluate the activity of HIV-1 IRES elements, we adopted dual luciferase reporter approach that combines an upstream RLUC and a downstream FLUC genes with 5'-UTR of HIV-1 mRNA. Using this dual-luciferase reporter construct, we showed that RACK1 inhibitor compounds efficiently inhibited IRES-mediated translation that utilitzed HIV-1 and HCV IRESes. We also observed inhibition of HIV-1 replication, both in one round infection and continuous replication assays. This inhibition was likely mediated through the inhibition of HIV-1 IRES-based translation. Thus, we concluded that IRES-mediated, non-classical pathway of viral mRNA translation was potentially contributing to HIV-1 protein synthesis and inhibiting this non-classical pathway can effectively inhibit the virus from replicating.

In the case of HIV-1, the role of IRES and RACK1 in viral replication is not fully understood. Several studies showed that translation initiation via IRES could be essential to HIV-1 replication since there are IRES-like structures located at the LTR and downstream from the gag initiation code $[36,37,42]$. This availability had encouraged us to analyze the role of IRES in HIV-1 replication and spread of the infection in the host cells. We propose that activation of HIV-1 IRES likely co-occurs with cell cycle arrest which may cause the start of the IRES based translation initiation. Here we present the evidence that inhibition of host's RACK1 reduces HIV-1 replication in human cell cultures. Also, we show that RACK1 inhibitors did efficiently inhibit HIV-1 replication in PBMCs and in monocyte-derived macrophages. Analyzing the effect of RACK1 inhibitors on viral proteins, we found out that p24 was reduced or absent in infected cells after treatment. Moreover, we used dualluciferase reporter constructs to show that RACK1 controls and affects IRES-mediated translation in human cells. Thus, we suggest that IRES-mediated, nonclassical, pathway of viral mRNA translation was essential for HIV-1 protein synthesis. Our findings demonstrate a specific function for ribosomal protein RACK1 in selective mRNA translation and establish the protein as a target for the development of broad antiviral intervention. However, it remains to be seen whether the compounds can have any adverse effect on the cellular IRES based translation of specific proteins.

Though most viruses are capable of translating their cap-dependent mRNAs, the capindependent mode of translation like IRES is reported to be used by diverse group of viruses under different physiological and environmental condition [53]. Polio virus, along with other picornaviruses, uses its proteases to cleave host eIF4G protein to cause a shutdown of host capdependent protein translation to facilitate its own cap-independent IRES driven mRNA translation initiation [54]. Though it was initially debated whether HIV-1 uses cap-independent IRES based translation initiation, expression of the structural protein Gag even when the cell is co-infected with polio virus indicated maintenance of cap-independent translation [55]. Subsequently, like in many other viruses, HIV-1 has also been found to initiate cap-independent translation under diverse physiological conditions like during oxidative and osmotic stress $[37,56]$, G2/M cell cycle arrest $[57$, 58], or when cap dependent translation proteins are cleaved by HIV-1 proteases [59-62]. While the early stages of HIV-1 protein synthesis are driven by a cap-dependent mechanism, the later time point viral protein synthesis switches to an IRES-mediated translation initiation model [36]. Here we show that host factor RACK1 is required for IRES driven translation in HIV-1 and report here the 
development of the functional inhibitor compounds for RACK1 that can show high efficiency in inhibiting IRES driven HIV-1 mRNA translation. This new class of compounds will be useful in not only dissecting the HIV-1 IRES based translation pathway studies but will also allow the use of the compounds as a durable anti-HIV-1 drug that may not become susceptible to the virus resistance that develop through mutation in the virus.

Noteworthy in the findings is the ability of the compounds to significantly inhibit the HCV IRES activities. As a member of the flaviviridae family, HCV replication is carried out by a capindependent mechanism mediated by the highly structured HCV IRES. Though at present several classes of effective, although expensive, drugs are available, availability of a new class of compounds that will be able to target the virus by inhibiting a key mechanism of HCV translation can be deemed as a significant advancement in the fight to cure HCV infection.

On the way to develop novel drugs to treat chronic complications of HIV-1 and HCV coinfection. The significance of this investigation lies in addressing an uncharacterized pathway of HIV replication and in its potential to identify novel antiviral therapeutic agents.

Author Contributions: Conceptualization, H.U. and S.D. ; methodology, M.B., S.N., S.D. and H.U; investigation, I.M., N.K., L.D.,; resources, A.I., L.D., M.B., S.N., S.D. and H.U.; data curation, H.U., writing - original draft preparation, H.U. and S.D.; writing- review and editing, M.B., S.N and H.U.; visualization, H.U.; supervision, M.B., S.N., and H.U.; funding acquisition, M.B., S.N, S.D. and H.U.

Funding: This research was funded by NIH Research Grants (1P50HL118006, 1R01HL125005 and U54MD007597), and the District of Columbia Developmental Center for AIDS Research grant (P30AI087714). The content is solely the responsibility of the authors and does not necessarily represent the official views of the National Institutes of Health.

- Acknowledgments: The author Sivanesan Dakshanamurthy wishes to acknowledge the support in part by DOD grant CA140882, CCSG grant NIH-P30 CA051008, GUMC Computational Chemistry Shared Resources (CCSR), and GUMC Lombardi Comprehensive Cancer Center. Dr. Wang Paul's lab at Howard University that is supported in part by the NIH/NIMHD G12MD007597 grant and the NIH/HICHD 1U54HD090257 grant has been instrumental in the luciferase assays.

Conflicts of Interest: The authors declare no conflict of interest. 


\section{References}

1. Islas-Flores, T.; Rahman, A.; Ullah, H.; Villanueva, M. A., The Receptor for Activated C Kinase in Plant Signaling: Tale of a Promiscuous Little Molecule. Front Plant Sci 2015, 6, 1090.

2. Adams, D. R.; Ron, D.; Kiely, P. A., RACK1, A multifaceted scaffolding protein: Structure and function. Cell Commun Signal 2011, 9, 22.

3. Kundu, N.; Dozier, U.; Deslandes, L.; Somssich, I. E.; Ullah, H., Arabidopsis scaffold protein RACK1A interacts with diverse environmental stress and photosynthesis related proteins. Plant Signal Behav 2013, 8, (5), e24012.

4. Gallo, S.; Ricciardi, S.; Manfrini, N.; Pesce, E.; Oliveto, S.; Calamita, P.; Mancino, M.; Maffioli, E.; Moro, M.; Crosti, M.; Berno, V.; Bombaci, M.; Tedeschi, G.; Biffo, S., RACK1 Specifically Regulates Translation through Its Binding to Ribosomes. Mol Cell Biol 2018, 38, (23).

5. Wolf, A. S.; Grayhack, E. J., Asc1, homolog of human RACK1, prevents frameshifting in yeast by ribosomes stalled at CGA codon repeats. RNA 2015, 21, (5), 935-45.

6. Ron, D.; Chen, C. H.; Caldwell, J.; Jamieson, L.; Orr, E.; Mochly-Rosen, D., Cloning of an intracellular receptor for protein kinase C: a homolog of the beta subunit of G proteins. Proc Natl Acad Sci U S A 1994, 91, (3), 839-43.

7. Chantrel, Y.; Gaisne, M.; Lions, C.; Verdiere, J., The transcriptional regulator Hap1p (Cyp1p) is essential for anaerobic or heme-deficient growth of Saccharomyces cerevisiae: Genetic and molecular characterization of an extragenic suppressor that encodes a WD repeat protein. Genetics 1998, 148, (2), 55969.

8. Ullah, H.; Scappini, E. L.; Moon, A. F.; Williams, L. V.; Armstrong, D. L.; Pedersen, L. C., Structure of a signal transduction regulator, RACK1, from Arabidopsis thaliana. Protein Sci 2008, 17, (10), 1771-80.

9. Majzoub, K.; Hafirassou, M. L.; Meignin, C.; Goto, A.; Marzi, S.; Fedorova, A.; Verdier, Y.; Vinh, J.; Hoffmann, J. A.; Martin, F.; Baumert, T. F.; Schuster, C.; Imler, J. L., RACK1 controls IRES-mediated translation of viruses. Cell 2014, 159, (5), 1086-1095.

10. Thompson, M. K.; Rojas-Duran, M. F.; Gangaramani, P.; Gilbert, W. V., The ribosomal protein Asc1/RACK1 is required for efficient translation of short mRNAs. Elife 2016, 5.

11. Thorslund, S. E.; Edgren, T.; Pettersson, J.; Nordfelth, R.; Sellin, M. E.; Ivanova, E.; Francis, M. S.; Isaksson, E. L.; Wolf-Watz, H.; Fallman, M., The RACK1 signaling scaffold protein selectively interacts with Yersinia pseudotuberculosis virulence function. PLoS One 2011, 6, (2), e16784.

12. Duff, D.; Long, A., Roles for RACK1 in cancer cell migration and invasion. Cell Signal 2017, 35, $250-255$.

13. Fan, Y.; Si, W.; Ji, W.; Wang, Z.; Gao, Z.; Tian, R.; Song, W.; Zhang, H.; Niu, R.; Zhang, F., Rack1 mediates Src binding to drug transporter P-glycoprotein and modulates its activity through regulating Caveolin-1 phosphorylation in breast cancer cells. Cell Death Dis 2019, 10, (6), 394.

14. Li, J. J.; Xie, D., RACK1, a versatile hub in cancer. Oncogene 2015, 34, (15), 1890-8.

15. Zhang, X.; Jain, R.; Li, G., Roles of Rack1 Proteins in Fungal Pathogenesis. Biomed Res Int 2016, $2016,4130376$.

16. Sartorello, R.; Amaya, M. J.; Nathanson, M. H.; Garcia, C. R., The plasmodium receptor for activated C kinase protein inhibits $\mathrm{Ca}(2+)$ signaling in mammalian cells. Biochem Biophys Res Commun 2009, 389, (4), 586-92.

17. Lin, W.; Zhang, Z.; Xu, Z.; Wang, B.; Li, X.; Cao, H.; Wang, Y.; Zheng, S. J., The association of receptor of activated protein kinase C 1 (RACK1) with infectious bursal disease virus viral protein VP5 and voltagedependent anion channel 2 (VDAC2) inhibits apoptosis and enhances viral replication. J Biol Chem 2015, 290, (13), 8500-10.

18. Bi, J.; Zhao, Q.; Zhu, L.; Li, X.; Yang, G.; Liu, J.; Yin, G., RACK1 is indispensable for porcine reproductive and respiratory syndrome virus replication and NF-kappaB activation in Marc-145 cells. Sci Rep 2018, 8, (1), 2985.

19. Zhang, C.; He, L.; Kang, K.; Chen, H.; Xu, L.; Zhang, Y., Screening of cellular proteins that interact with the classical swine fever virus non-structural protein 5 A by yeast two-hybrid analysis. J Biosci 2014, 39, (1), 63 74.

20. Demirov, D.; Gabriel, G.; Schneider, C.; Hohenberg, H.; Ludwig, S., Interaction of influenza A virus matrix protein with RACK1 is required for virus release. Cell Microbiol 2012, 14, (5), 774-89.

21. Jia, B.; Guo, M.; Li, G.; Yu, D.; Zhang, X.; Lan, K.; Deng, Q., Hepatitis B virus core protein sensitizes hepatocytes to tumor necrosis factor-induced apoptosis by suppression of the phosphorylation of mitogenactivated protein kinase kinase 7. J Virol 2015, 89, (4), 2041-51.

22. Johnson, A. G.; Grosely, R.; Petrov, A. N.; Puglisi, J. D., Dynamics of IRES-mediated translation. Philos Trans $R$ Soc Lond B Biol Sci 2017, 372, (1716).

23. Pelletier, J.; Sonenberg, N., Internal initiation of translation of eukaryotic mRNA directed by a sequence derived from poliovirus RNA. Nature 1988, 334, (6180), 320-5.

24. Jang, S. K.; Krausslich, H. G.; Nicklin, M. J.; Duke, G. M.; Palmenberg, A. C.; Wimmer, E., A segment of the 5 ' nontranslated region of encephalomyocarditis virus RNA directs internal entry of ribosomes during in vitro translation. J Virol 1988, 62, (8), 2636-43.

25. Jackson, R. J., The current status of vertebrate cellular mRNA IRESs. Cold Spring Harb Perspect Biol 2013, 5, (2). 
26. Cherry, S.; Doukas, T.; Armknecht, S.; Whelan, S.; Wang, H.; Sarnow, P.; Perrimon, N., Genome-wide RNAi screen reveals a specific sensitivity of IRES-containing RNA viruses to host translation inhibition. Genes Dev 2005, 19, (4), 445-52.

27. Martins, N.; Imler, J. L.; Meignin, C., Discovery of novel targets for antivirals: learning from flies. Curr Opin Virol 2016, 20, 64-70.

28. Hafirassou, M. L.; Meignin, C.; Baumert, T.; Schuster, C., [From fly to man: RACK1, an essential actor of the dependent viral translation of IRES]. Med Sci (Paris) 2015, 31, (5), 469-72.

29. Lee, J. S.; Tabata, K.; Twu, W. I.; Rahman, M. S.; Kim, H. S.; Yu, J. B.; Jee, M. H.; Bartenschlager, R.; Jang, S. K., RACK1 mediates rewiring of intracellular networks induced by hepatitis C virus infection. PLoS Pathog 2019, 15, (9), e1008021.

30. Nakagawa, K.; Lokugamage, K. G.; Makino, S., Viral and Cellular mRNA Translation in CoronavirusInfected Cells. Adv Virus Res 2016, 96, 165-192.

31. Liu, D. X.; Inglis, S. C., Internal entry of ribosomes on a tricistronic mRNA encoded by infectious bronchitis virus. J Virol 1992, 66, (10), 6143-54.

32. Thiel, V.; Siddell, S. G., Internal ribosome entry in the coding region of murine hepatitis virus mRNA $5 . J$ Gen Virol 1994, 75 ( Pt 11), 3041-6.

33. Jendrach, M.; Thiel, V.; Siddell, S., Characterization of an internal ribosome entry site within mRNA 5 of murine hepatitis virus. Arch Virol 1999, 144, (5), 921-33.

34. Gallina, A.; Rossi, F.; Milanesi, G., Rack1 binds HIV-1 Nef and can act as a Nef-protein kinase C adaptor. Virology 2001, 283, (1), 7-18.

35. Buck, C. B.; Shen, X.; Egan, M. A.; Pierson, T. C.; Walker, C. M.; Siliciano, R. F., The human immunodeficiency virus type 1 gag gene encodes an internal ribosome entry site. J Virol 2001, 75, (1), 18191.

36. Amorim, R.; Costa, S. M.; Cavaleiro, N. P.; da Silva, E. E.; da Costa, L. J., HIV-1 transcripts use IRESinitiation under conditions where Cap-dependent translation is restricted by poliovirus 2A protease. PLoS One 2014, 9, (2), e88619.

37. Gendron, K.; Ferbeyre, G.; Heveker, N.; Brakier-Gingras, L., The activity of the HIV-1 IRES is stimulated by oxidative stress and controlled by a negative regulatory element. Nucleic Acids Res 2011, 39, (3), 902-12.

38. Plank, T. D.; Whitehurst, J. T.; Cencic, R.; Pelletier, J.; Kieft, J. S., Internal translation initiation from HIV-1 transcripts is conferred by a common RNA structure. Translation (Austin) 2014, 2, (1), e27694.

39. Sabila, M.; Kundu, N.; Smalls, D.; Ullah, H., Tyrosine Phosphorylation Based Homo-dimerization of Arabidopsis RACK1A Proteins Regulates Oxidative Stress Signaling Pathways in Yeast. Front Plant Sci 2016, 7, 176 .

40. Ullah, H.; Hou, W.; Dakshanamurthy, S.; Tang, Q., Host targeted antiviral (HTA): functional inhibitor compounds of scaffold protein RACK1 inhibit herpes simplex virus proliferation. Oncotarget 2019, 10, (35), 3209-3226.

41. Kumari, N.; Iordanskiy, S.; Kovalskyy, D.; Breuer, D.; Niu, X.; Lin, X.; Xu, M.; Gavrilenko, K.; Kashanchi, F.; Dhawan, S.; Nekhai, S., Phenyl-1-Pyridin-2yl-ethanone-based iron chelators increase IkappaB-alpha expression, modulate CDK2 and CDK9 activities, and inhibit HIV-1 transcription. Antimicrobial agents and chemotherapy 2014, 58, (11), 6558-71.

42. Plank, T. D.; Whitehurst, J. T.; Kieft, J. S., Cell type specificity and structural determinants of IRES activity from the 5' leaders of different HIV-1 transcripts. Nucleic Acids Res 2013, 41, (13), 6698-714.

43. Carvajal, F.; Vallejos, M.; Walters, B.; Contreras, N.; Hertz, M. I.; Olivares, E.; Caceres, C. J.; Pino, K.; Letelier, A.; Thompson, S. R.; Lopez-Lastra, M., Structural domains within the HIV-1 mRNA and the ribosomal protein S25 influence cap-independent translation initiation. FEBS J 2016, 283, (13), 2508-27.

44. Mokrejs, M.; Vopalensky, V.; Kolenaty, O.; Masek, T.; Feketova, Z.; Sekyrova, P.; Skaloudova, B.; Kriz, V.; Pospisek, M., IRESite: the database of experimentally verified IRES structures (www.iresite.org). Nucleic Acids Res 2006, 34, (Database issue), D125-30.

45. Locker, N.; Chamond, N.; Sargueil, B., A conserved structure within the HIV gag open reading frame that controls translation initiation directly recruits the 40 S subunit and eIF3. Nucleic Acids Res 2011, 39, (6), 2367 77.

46. Barrera, A.; Olguin, V.; Vera-Otarola, J.; Lopez-Lastra, M., Cap-independent translation initiation of the unspliced RNA of retroviruses. Biochim Biophys Acta Gene Regul Mech 2020, 1863, (9), 194583.

47. Martinez-Salas, E.; Francisco-Velilla, R.; Fernandez-Chamorro, J.; Embarek, A. M., Insights into Structural and Mechanistic Features of Viral IRES Elements. Front Microbiol 2017, 8, 2629.

48. Yamamoto, H.; Collier, M.; Loerke, J.; Ismer, J.; Schmidt, A.; Hilal, T.; Sprink, T.; Yamamoto, K.; Mielke, T.; Burger, J.; Shaikh, T. R.; Dabrowski, M.; Hildebrand, P. W.; Scheerer, P.; Spahn, C. M., Molecular architecture of the ribosome-bound Hepatitis C Virus internal ribosomal entry site RNA. EMBO J 2015, 34, (24), 3042-58.

49. Quade, N.; Boehringer, D.; Leibundgut, M.; van den Heuvel, J.; Ban, N., Cryo-EM structure of Hepatitis C virus IRES bound to the human ribosome at 3.9-A resolution. Nat Commun 2015, 6, 7646.

50. Yokoyama, T.; Machida, K.; Iwasaki, W.; Shigeta, T.; Nishimoto, M.; Takahashi, M.; Sakamoto, A.; Yonemochi, M.; Harada, Y.; Shigematsu, H.; Shirouzu, M.; Tadakuma, H.; Imataka, H.; Ito, T., HCV IRES Captures an Actively Translating 80S Ribosome. Mol Cell 2019, 74, (6), 1205-1214 e8. 
51. des Georges, A.; Dhote, V.; Kuhn, L.; Hellen, C. U.; Pestova, T. V.; Frank, J.; Hashem, Y., Structure of mammalian eIF3 in the context of the $43 S$ preinitiation complex. Nature 2015, 525, (7570), 491-5.

52. Chamond, N.; Locker, N.; Sargueil, B., The different pathways of HIV genomic RNA translation. Biochemical Society transactions 2010, 38, (6), 1548-52.

53. Hanson, P. J.; Zhang, H. M.; Hemida, M. G.; Ye, X.; Qiu, Y.; Yang, D., IRES-Dependent Translational Control during Virus-Induced Endoplasmic Reticulum Stress and Apoptosis. Front Microbiol 2012, 3, 92.

54. Etchison, D.; Milburn, S. C.; Edery, I.; Sonenberg, N.; Hershey, J. W., Inhibition of HeLa cell protein synthesis following poliovirus infection correlates with the proteolysis of a 220,000-dalton polypeptide associated with eucaryotic initiation factor 3 and a cap binding protein complex. J Biol Chem 1982, 257, (24), 14806-10.

55. Monette, A.; Valiente-Echeverria, F.; Rivero, M.; Cohen, E. A.; Lopez-Lastra, M.; Mouland, A. J., Dual mechanisms of translation initiation of the full-length HIV-1 mRNA contribute to gag synthesis. PLoS One 2013, 8, (7), e68108.

56. Monette, A.; Ajamian, L.; Lopez-Lastra, M.; Mouland, A. J., Human immunodeficiency virus type 1 (HIV1) induces the cytoplasmic retention of heterogeneous nuclear ribonucleoprotein A1 by disrupting nuclear import: implications for HIV-1 gene expression. J Biol Chem 2009, 284, (45), 31350-62.

57. Vallejos, M.; Deforges, J.; Plank, T. D.; Letelier, A.; Ramdohr, P.; Abraham, C. G.; Valiente-Echeverria, F.; Kieft, J. S.; Sargueil, B.; Lopez-Lastra, M., Activity of the human immunodeficiency virus type 1 cell cycledependent internal ribosomal entry site is modulated by IRES trans-acting factors. Nucleic Acids Res 2011, 39, (14), 6186-200.

58. Brasey, A.; Lopez-Lastra, M.; Ohlmann, T.; Beerens, N.; Berkhout, B.; Darlix, J. L.; Sonenberg, N., The leader of human immunodeficiency virus type 1 genomic RNA harbors an internal ribosome entry segment that is active during the G2/M phase of the cell cycle. J Virol 2003, 77, (7), 3939-49.

59. Alvarez, E.; Castello, A.; Menendez-Arias, L.; Carrasco, L., HIV protease cleaves poly(A)-binding protein. Biochem J 2006, 396, (2), 219-26.

60. Castello, A.; Franco, D.; Moral-Lopez, P.; Berlanga, J. J.; Alvarez, E.; Wimmer, E.; Carrasco, L., HIV- 1 protease inhibits Cap- and poly(A)-dependent translation upon eIF4GI and PABP cleavage. PLoS One 2009, 4, (11), e7997.

61. Ohlmann, T.; Prevot, D.; Decimo, D.; Roux, F.; Garin, J.; Morley, S. J.; Darlix, J. L., In vitro cleavage of eIF4GI but not eIF4GII by HIV-1 protease and its effects on translation in the rabbit reticulocyte lysate system. J Mol Biol 2002, 318, (1), 9-20.

62. Perales, C.; Carrasco, L.; Ventoso, I., Cleavage of eIF4G by HIV-1 protease: effects on translation. FEBS Lett 2003, 533, (1-3), 89-94. 